Horizons philosophiques

\title{
Une constellation et un météore dans un ciel hyperboréen ou Herder et Thorild sur la théorie des climats de Montesquieu
}

\section{Roch Duval}

Volume 13, numéro 2, printemps 2003

Herder (1744-1803) : le clair-obscur

URI : https://id.erudit.org/iderudit/801238ar

DOI : https://doi.org/10.7202/801238ar

Aller au sommaire du numéro

Éditeur(s)

Collège Édouard-Montpetit

ISSN

1181-9227 (imprimé)

1920-2954 (numérique)

Découvrir la revue

Citer cet article

Duval, R. (2003). Une constellation et un météore dans un ciel hyperboréen ou Herder et Thorild sur la théorie des climats de Montesquieu. Horizons

philosophiques, 13(2), 69-92. https://doi.org/10.7202/801238ar d'utilisation que vous pouvez consulter en ligne. 


\title{
UNE CONSTELLATION ET UN MÉTÉORE DANS UN CIEL HYPERBORÉEN OU \\ HERDER ET THORILD SUR LA THÉORIE DES CLIMATS DE MONTESQUIEU
}

\author{
Ce noble esprit a été méconnu par des époques et \\ des partis opposés, un peu par sa faute pourtant, car il \\ avait pour défaut de n'être pas une étoile de premier \\ ou de second plan, mais un faisceau d'étoiles, \\ où chacun ensuite épelle telle ou telle constellation. \\ Jean-Paul1 \\ Der låg en verld $i$ Kaos i hans hjema, men sol sken \\ icke, icke morgonstjerna, en Meteor allen som \\ knallade och sprang. \\ Esaias Tegnér?
}

L'implication de Herder dans l'émergence du courant littéraire allemand désigné par l'expression Sturm und Drang (Tempête et Élan) n'est plus à démontrer. Or, à quelques années de différence près, l'existence d'un mouvement similaire en Suède demeure un fait méconnu de la majorité des spécialistes francophones. Par conséquent, il se révèle important de mentionner l'existence de la variante suédoise de ce mouvement philosophico-littéraire si l'on tient particulièrement compte du fait que la philosophie de Thomas Thorild, sa figure de proue, présente à plusieurs égards une étonnante ressemblance avec celle de Herder. Cependant, une telle filiation philosophique, aussi méconnue soit-elle, n'échappa pas à l'esprit sagace du philosophe allemand Ernst Cassirer. En effet, dans une série d'articles fouillés, le philosophe natif de Breslau (aujourd'hui Wroclaw) s'est ingénié à retracer l'influence de Herder dans les écrits philosophiques de Thorild. La conclusion de Cassirer est péremptoire : l'influence de Herder sur Thorild se serait fait sentir dès la parution du petit texte "Vom Erkennen und Empfinden der 
menschlichen Seele»(1778). Dans cet article, je tente d'évaluer la pertinence de la conclusion à laquelle est parvenu Cassirer en comparant les positions de Herder et de Thorild sur un point précis et qui n'apparaît pas dans l'argument de Cassirer. Ainsi, l'examen de leur attitude respective quant à la théorie des climats de Montesquieu sera l'élément de comparaison retenu et nous permettant d'évaluer le bien-fondé de la thèse avancée par le philosophe allemand. Les éléments ainsi dégagés nous incitent à réévaluer non seulement la conclusion de Cassirer mais également à proposer une autre interprétation quant à la prétendue ressemblance entre le Sturm und Drang allemand et son équivalent suédois.

\section{La thèse de Cassirer}

En 1799, un livre intitulé Maximum seu Archimetria stupéfiait l'univers intellectuel allemand. Non seulement un auteur - sous le couvert de l'anonymat de surcroît - osait rompre en visière avec un des monuments les plus respectés de la philosophie allemande (et, par ricochet, avec tout le programme de l'Aufklärung), soit la Critique de la raison pure d'Emmanuel Kant, mais on s'étonna davantage lorsque l'on se rendit compte, après quelques mois, que la critique qui était adressée à Kant ressemblait singulièrement à une autre critique, celle contenue dans la Metakritik zur Kritik der reinen Vernunft (1799) de Johann Gottried Herder. Véritable coïncidence ou plutôt parfaite synchronie dans le monde des idées, le Maximum seu Archimetria était apparu pratiquement au même moment et, selon toute vraisemblance, indépendamment de la célèbre critique de Herder ${ }^{3}$. Cette singularité n'échappa pas au philosophe allemand car celui-ci se mit aussitôt en quête de découvrir l'identité de ce mystérieux auteur, à la fois pour assouvir sa curiosité et pour manifester à celui-ci la très haute opinion qu'il s'était faite de ce livre. Caroline - épouse toute dévouée de Herder - rapportait d'ailleurs dans une lettre adressée à Johann Wilhelm Ludwig Gleim (1719-1803) toute l'admiration que vouait son mari à ce texte : «Mein Mann hält das lateinische Buch sehr hoch; er liest jeden Tag darinnen" 4 En dépit de l'accueil peu chaleureux que la critique avait réservée à l'Archimetria, 5 Herder publia le 6 octobre 1800 une analyse très élogieuse du mystérieux livre dans l'Erfurter Nachrichten 6 . Entre temps, l'énigmatique auteur s'était manifesté à Herder. II s'agissait d'un poète et philosophe suédois, expatrié depuis 1793 , et œuvrant à titre de bibliothécaire et de professeur à l'Université de Greifswald depuis 1796. Dans une 
lettre adressée à Herder, en date du 23 février 1800, Thomas Thorild - car tel est son nom - se présente en précisant qu'il fut le premier à introduire en Suède une poésie tirant son inspiration de celle en vers libres de Friedrich Gottlieb Klopstock (1724-1803) ainsi que celle du britannique Edward Young (1683-1765) :

Der Verfasser jener Archimetrie ist Thomas Thorild, schwedischer Dichter, und in seiner Vaterland der erste Anstimmer des Youngisch-Klopstockischen Tons 7 .

II est assez significatif que Thorild se présente à Herder en faisant précisément valoir qu'il se perçoit davantage comme un poète que comme un philosophe au sens strict du terme. Cette singularité préoccupera d'ailleurs un grand nombre de philosophes ou d'historiens de la philosophie qui se sont abondamment interrogés sur la pertinence, ou simplement sur le bien-fondé, d'inclure Herder ou Thorild parmi des philosophes 8 . Notons d'ailleurs, à l'époque où il vivait encore en Suède, que Thorild avait pris l'habitude de se désigner comme un autorn. Cela se révèle particulièrement intéressant car, comme on le sait, la langue suédoise possède deux termes pour désigner un "auteur". Nous pouvons aussi bien utiliser le terme författare que le terme upphovsman, mais le mot autorn n'existe pas. II est tout juste permis d'utiliser la forme "auktor»qui dérive de l'anglais "author»9. Or, en créant le néologisme autorn - forme la plus anglicisée qu'il soit possible d'utiliser - Thorild désire manifestement signaler la dette qu'il a envers la philosophie anglaise et plus particulièrement envers Lord Shaftesbury ${ }^{10}$. Cela se révèle digne de mention dans la mesure où Herder se percevait également comme un Dichterphilosoph. Voilà un élément particulièrement intéressant laissant poindre une parenté profonde, non seulement dans le dessein philosophique mais également dans le "style» littéraire de Thorild et Herder. Que penser de ces coïncidences?

Bien que le nom de Thomas Thorild soit parfois mentionné dans certains livres spécialisés retraçant patiemment l'histoire de la philosophie kantienne, force est de constater qu'il demeure néanmoins une figure honteusement méconnue hors de la Suède. Cependant, au fil des ans, ses compatriotes suédois lui ont consacré bon nombre d'études ${ }^{11}$. Par contre, il revient à Ernst Cassirer d'avoir tenté plus que quiconque de démontrer la profonde parenté unissant la philosophie de Thorild à celle de Herder. ${ }^{12}$ Ainsi, lors de son exil forcé en Suède - où il séjourna à Göteborg de 1935 à 1941 — 
Cassirer publia deux études où il s'efforce de comparer systématiquement les philosophies respectives de Thorild et de Herder ${ }^{13}$. II existe, bien entendu, la singulière similitude, déjà notée, dans la visée théorique de l'Archimetria et celle de la Metakritik zur Kritik der reinen Vernunft, mais Cassirer ne compulsa pas uniquement les écrits de Thorild rédigés lors de son exil à Greifswald ${ }^{14}$. II scruta également l'ensemble de la vaste production littéraire du philosophe suédois. Par voie de conséquence, un examen approfondi des textes de Thorild lui permet d'énoncer une thèse selon laquelle l'influence de Herder s'est fait sentir très tôt chez le Suédois. En effet, Cassirer soutient que même si les spécialistes suédois ont parfois vu juste en notant au passage - mais d'une façon diffuse et hélas trop souvent anecdotique - l'influence de la philosophie spinoziste tant chez Thorild que chez Herder ${ }^{15}$ c'est davantage dans un texte de Herder intitulé "Vom Erkennen und Empfinden der menschlichen Seele»(1778) qu'il faut chercher ce qui les unit et les rapproche. Même s'il ne possède pas de preuves directes pour étayer ce qu'il avance, 16 Cassirer croit que la clé du mystère expliquant la proche parenté caractérisant leur critique de la philosophie kantienne réside dans ce petit texte de Herder; texte qui aurait influencé Thorild dès son tout jeune âge.

Sans exposer davantage la thèse de Cassirer, qui au demeurant démontre un intérêt évident, je propose de mettre celle-ci à l'épreuve en soumettant un texte de Thorild, laissé pour compte - il me semble - dans l'analyse de Cassirer. Le texte en question s'intitule Critik öfver Montesquieu (1788) ${ }^{17}$. L'intérêt que suscite ce petit texte est multiple. D'une part, la période où il fut rédigé se situe à mi-chemin entre les deux périodes amplement analysées par Cassirer, soit 1778 (période formatrice de Lund pour Thorild) et 1799 (période de la maturité et de l'exil à Greifswald), alors que le texte sur Montesquieu est à la fois caractéristique des années 80 (période du Sturm und Drang suédois et de la renommée littéraire) et de son séjour dans la capitale suédoise. Ainsi, tant par l'époque que par le lieu, ce texte diffère de ceux utilisés par Cassirer pour établir sa thèse. D'autre part, Critik öfver Montesquieu permet de comparer les positions philosophiques de Thorild et de Herder, non pas in abstracto, mais bien in concreto, car ce qui est immédiatement comparé c'est l'attitude (critique ou pas) qu'ils adoptent envers un seul et même auteur, soit Montesquieu. Ainsi, si la thèse de Cassirer se révélait véridique, alors il faudrait postuler dès le départ que Thorild et Herder adoptent la même attitude face à Montesquieu. Car, si cela n'était pas le cas, comment 
alors expliquer une adéquation au niveau des idées entre Thorild et Herder en 1778, puis en 1799, mais non en 1788. En d'autres termes, si - comme le soutient Cassirer - Thorild était déjà imprégné des thèses de Herder en 1778 et que cette influence s'est maintenue nolens volens (mais en empruntant. il va de soi, des chemins différents) jusqu'en 1799, alors nous devrions être en mesure de vérifier s'il existe également une adéquation au niveau des idées à une période intermédiaire. Thorild ne s'étant jamais prononcé ouvertement contre les thèses de Herder, il faut donc en conclure, si l'on suit le raisonnement de Cassirer, qu'ils devraient avoir la même opinion sur la théorie des climats de Montesquieu. Somme toute, seul un examen, si sommaire soit-il, des positions respectives des auteurs concernés pourra nous fournir un élément de réponse.

II s'agit donc, en clair, d'évaluer la thèse de Cassirer en soumettant celle-ci à l'examen des théories respectives de Thorild et de Herder en ce qui concerne la théorie des climats de Montesquieu. Si nous sommes en mesure de démontrer une similitude au niveau des attitudes théoriques chez Thorild et Herder relativement à cet aspect de la théorie de Montesquieu, alors nous pourrons croire que la thèse de Cassirer se révèle fondée ou du moins corroborée. Cependant, si cela n'est pas le cas, alors il faudra s'interroger sur la pertinence de celle-ci, voire proposer une autre explication. II s'agit donc, dans un premier temps, d'exposer le cheminement intellectuel de Thorild et de mentionner à quel moment ce dernier questionne la théorie des climats de Montesquieu et, partant, quelle utilisation il en fait. Dans un second temps on tentera de localiser dans des textes de Herder de la même époque l'usage (ou la mention) qui est fait de la thèse de la théorie des climats. Doté de tels éléments, il nous sera possible de comparer les positions respectives de l'un et de l'autre quant à la théorie des climats de Montesquieu et, subséquemment, nous prononcer sur la validité de l'hypothèse de Cassirer..

\section{Thorild et la théorie des climats de montesquieu.}

Bien qu'il soit méconnu de la plupart des philosophes ne connaissant ni le suédois ou l'allemand, Thomas Thorild représente une figure incontournable de l'histoire de la littérature et de la philosophie en Suède. Sa renommé, d'une part, découle du climat de polémique et de scandale qu'évoque son nom. En effet, comment ne pas oublier son interminable querelle, déchaînant toutes les passions ${ }^{18}$, avec le très respectable Johan Henric Kellgren (1751-1795) ${ }^{19}$ puis, prenant la 
relève, avec l'acharné et opiniâtre Karl Gustaf av Leopold (1756$1829)^{20}$, tous deux fiers représentants suédois du Classicisme français ${ }^{21}$ et entièrement dévoués au roi Gustave III (1746-1792). D'autre part, comme nous l'avons mentionné auparavant, c'est à Thorild qu'il faut entièrement attribuer l'existence sur le sol suédois du mouvement littéraire Sturm und Drang22. Le rejet du modèle du Classicisme français où prédomine une conception toute française $d u$ goût qu'il exècre, l'introduction des vers libres qu'il hérite de Klopstock, l'appel fait à la poésie nationaliste de Young où on peut laisser libre cours au génie naturel23, la sympathie qu'il témoigne à l'endroit de la philosophie (des sentiments) de Jean-Jacques Rousseau, voilà tout ce qui fait de lui un être original, mais également marginal24 dans une Suède où règne sans merci un roi entièrement vendu à la cause française. Avant d'aborder directement le problème qui nous concerne en plein, je considère opportun de retracer les étapes les plus importantes du développement personnel et intellectuel de Thomas Thorild afin de cerner davantage le personnage et de situer en contexte sa compréhension de la théorie des climats de Montesquieu..

Thomas Jönsson Thorén 25 est né le 18 avril 1759 à Svarteborg26. Ses parents meurent très tôt et, en 1765, il est envoyé chez son frère Olof (alors âgé de 21 ans) à Kungälv afin d'entreprendre son éducation primaire. Mais un drame frappe de nouveau la famille du jeune Thomas lorsque son frère Olof meurt à l'âge de 26 ans. Heureusement son maître de classe, le directeur d'école Anders Tranchell, le recueille chez lui, ce qui permet à Thorild de terminer ses études primaires. Cet événement fut déterminant dans la vie de Thorild car Tranchell, en plus de lui donner le confort d'un foyer, lui offrit un milieu de vie où les livres (et l'éducation) avaient une grande valeur. "Vistelsen i rektorshemmet har säkerligen varit av betydelse för Thorilds intellektuella utveckling och för hans inriktning mot studier27». Puis, à l'âge de $121 / 2$, il se dirige vers Göteborg pour entreprendre ses études secondaires. Là encore Anders Tranchell intervient en sa faveur d'une façon providentielle, car il le met en contact avec son frère Jonas (1740), caissier bien nanti à la Compagnie des Indes Orientales. L'ascendant de ce dernier est tel que lorsque Thorild parle de lui, il le désigne affectueusement en utilisant l'épithète «min välgörare», mon bienfaiteur. Le séjour de Thorild à Göteborg se poursuit jusqu'en 1775. C'est là que l'un de ses professeurs, Johan Gothenius ${ }^{28}$, l'initie à la poésie d'Ossian; poésie qui, comme on le 
sait, est indissociable du mouvement du Sturm und Drang en Allemagne. Au terme de ses études à Göteborg, il arrive à Lund, le 22 septembre 1775 et il s'inscrit à la Faculté de droit. On sait toutefois qu'il y demeure uniquement une session, préférant retourner à Göteborg en juillet 1776.29 Puis, en octobre 1777, il retourne de nouveau à Lund où il reprend à la fois ses études en droit à l'université et entre au service de la famille du magistrat et commerçant Olof Liljewalch comme précepteur; poste qu'il occupera jusqu'au début de l'automne 1779. Sur un plan intellectuel, son second séjour fut des plus profitables.

Det är under sin studenttid i Lund han gör bekantskap med de nya, delvis revolutionära andliga strömningarna ute i Europa, i Frankrike, England och Tyskland. När han i slutet av 1780 lämnar universitetsstaden har han i bagaget en ny, kristendomsfientlig livsäskädning, nya filosofiska grundtankar och en ny, Werther-influerad syn på litteraturen. (...) Sin Lunda-tid har han använt främst till sina egna märkligt fria studierpo.

En effet, c'est à Lund que survient véritablement l'éveil intellectuel du jeune Suédois. En premier lieu Thorild abjure sa foi dans la religion chrétienne ${ }^{31}$. Puis, découvrant la philosophie spinoziste, ainsi que celle des membres de l'aile marchante des Lumières en France (Condillac, Helvétius, Holbach), il élabore une théorie philosophique où se mêlent diverses influences, parmi lesquelles on peut nommer le panthéisme, le matérialisme et le sensualisme. La lecture de l'Émile de Rousseau eut également une influence considérable sur son développement intellectuel. Combinant toutes ces influences (mais sans toutefois sombrer dans un éclectisme plat), Thorild énonce une théorie originale se caractérisant comme une sorte de panthéisme matérialiste, théorie dans laquelle le terme kraft (force) est le maître mot. Mais se sentant tout de même à l'étroit à Lund 32 - où même la nature lui semble si peu suédoise, si différente de celle qu'il a connue et tant appréciée dans son patelin natal - il mûrit l'ambitieux projet d'aller tenter sa chance dans la capitale suédoise, Stockholm.

Quittant Lund peu de temps avant la fête de Noël de l'année 1780, le jeune Thomas se dirige vers Stockholm où il arrive le 17 février 1781. Le 10 avril 1781, soit à peine deux mois après son arrivée dans la capitale, il est accueilli à Kungsholmen ${ }^{33}$ par la famille de son vieil ami Arvid Hindström. II est intéressant de noter que 
Thorild retrouve dans ce nouvel environnement, judicieusement situé sur les rives du splendide lac Mälaren, une nouvelle occasion de renouer avec le plaisir esthétique découlant d'une contemplation de la nature et des paysages qu'il décrit comme typiquement suédois. Le 28 mai 1781, il écrit à son ami Heurlin : «I Fridens och Landets sköte, sitter jag vid mit öpna fönster och skrifver till dig, och drikker med alla mina Sinnen Guds Skönhet, och andas hans Väsende34». Outre la redécouverte de l'esthétique des paysages suédois, le séjour à Kungsholmen retient l'attention des spécialistes, car c'est précisément à cet endroit que se révèle au grand jour le génie poétique de Thorild. Ainsi, c'est dans le chef-d'œuvre Passionerna, pièce maîtresse du Sturm und Drang suédois, que se condensent toutes les influences qu'il a reçues à Stockholm. La parution du poème Passionerna, que Thorild avait soumis à un concours littéraire organisé par la société littéraire Utile Dulci, présidée à cette époque par Kellgren, déclencha une célèbre querelle entre le représentant du Sturm und Drang et le gardien du bon goût français, Kellgren. On reprochait principalement à Thorild d'avoir introduit dans la poésie suédoise l'hexamètre et les vers libres, deux entorses impardonnables au bon goût français. Son audace lui valut d'ailleurs d'innombrables désagréments, ses adversaires le taxant même à l'occasion d'être insensé ou tout simplement fou (vansinnig) ${ }^{35}$.

Se sentant de plus en plus à l'étroit et incompris en Suède, Thorild rêve de contrées étrangères où, croit-il, il pourra s'épanouir et satisfaire sa soif de liberté et côtoyer des esprits éclairés. L'Angleterre, terre par excellence de réformes sociales et de liberté, capte particulièrement son attention. Or, pour s'offrir le luxe d'y vivre autrement que comme un vulgaire paria, il doit au préalable obtenir un doctorat s'il ne veut pas être perçu comme un aventurier insignifiant. C'est donc dans cette optique qu'en mars 1787 il se dirige vers Uppsala afin de compléter ses études en droit. Le soir de Pâques de l'année 178836, devant une salle comble et en présence du roi Gustave III37 que l'on dit soucieux de rencontrer ce brillant esprit, l'auteur des Passionernas commente avec fougue certaines thèses de Montesquieu. Compte tenu du climat intellectuel qui régnait en Suède à cette époque, l'exercice n'a rien d'anodin. Qu'est-ce à dire?

En effet, il faut se rappeler qu'à cette époque en Suède, tout comme dans le reste de l'Europe d'ailleurs, Montesquieu jouissait d'un certain prestige et la théorie des climats, en particulier, y était particulièrement bien accueillie ${ }^{38}$. Profitons de l'occasion qui nous est 
offerte pour présenter certains éléments spécifiques afin de caractériser l'accueil qui fut réservé à la théorie des climats en Suède. Disons d'entrée de jeu qu'avant même que les théories de Montesquieu sur le climat ne soient diffusées dans cette contrée nordique, il y existait déjà une importante réflexion philosophique et/ou scientifique sur les phénomènes climatiques. Considérant cela, il n'est guère étonnant de retrouver dans notre vie quotidienne le nom d'un Suédois, Anders Celsius (1701-1744), associé à la mesure scientifique de la température. Ce fait dénote l'intérêt indéfectible des peuples nordiques pour l'étude (sous toutes ses formes) des phénomènes climatiques et plus particulièrement de l'effet (bénéfique) du climat nordique sur la constitution tant physique que morale des individus y subissant son influence. En effet, dès le milieu du XVle siècle se dessine avec Olaus Magnus (1490-1544) une vision plus positive des contrées nordiques que celle véhiculée par la théorie antique des climats. II faut $s e$ rappeler que dans la théorie antique des climats, tant chez Hippocrate ${ }^{39}$ que chez Aristote ${ }^{40}$, les contré nordiques (à l'instar des contrées tropicales) étaient essentiellement perçues comme des zones dégénérées et peu propices à l'éclosion d'une vie saine et équilibrée, caractéristique des zones tempérées. La zone tempérée, coextensive au site de la civilisation grecque, se caractérisait favorablement comme une zone idéale profitant à la fois des avantages associés aux zones extrêmes - soit, d'une part, le goût de liberté et la vivacité des peuples nordiques et, d'autre part, l'intelligence et la vivacité d'esprit des asiatiques - et échappant aux vices également attribuées à ces zones extrêmes - bêtise des peuples nordiques et paresse et couardise des asiatiques. Somme toute, la zone tempérée était perçue comme favorisée et plus équilibrée que les zones extrêmes, lesquelles étaient qualifiées de dégénérées ${ }^{41}$. En somme, une telle théorie était en quelque sorte utilisée pour laisser croire aux habitants des zones tempérées qu'ils étaient supérieurs et dotés de meilleurs attributs (tant physiques que mentaux) que les habitants des zones extrêmes. Manifestation à peine déguisée d'un eurocentrisme 42 insolent, la théorie antique des climats connaît dans la France du XVIIe siècle une certaine popularité auprès du père jésuite Dominique Bouhours (1632-1702). Au début du XVIIle siècle, le relais est activement pris par l'abbé J. B. Dubos qui dans les Réflexions critiques sur la poésie et la peinture ${ }^{43}$ consolide les préjugés "eurocentristes»en tentant d'établir une (grossière) adéquation entre le climat clément régnant sur la France, l'Italie et la 
Grèce et la qualité exceptionnelle de leurs créations artistiques, critères absolus du bon goût 44 .

Or, en marge de cet eurocentrisme "restreint», une vision plus positive du Nord se dessine avec Ericus Olai, (1550-1627) à la fin du $X V$ e siècle, et dès le milieu du XVle siècle Olaus Magnus, dans Historia om de nordiska folken 45 (Histoire des peuples nordiques) peut soutenir que le climat nordique fortifie les gens qui y vivent en les rendant non seulement braves mais également industrieux. Dès lors le climat septentrional cesse d'être perçu comme un handicap, mais acquiert une valeur positive en conférant des attributs désirables aux êtres qui subissent son influence. Le mouvement de revalorisation des contrées nordiques, que l'on appelle goticisme (göticism en suédois), venait d'éclore. Dans la foulée de ce mouvement de revalorisation des contrées nordiques - allant de pair avec un mouvement de dénigrement des pays méridionaux - il faut absolument inclure Olof Rudbeck (1630-1702), auteur du célèbre Atlantica (1679-1702) dans lequel il écrit que les Goths «étaient à la fois plus grands et plus courageux de corps et d'esprit» 46 que les gens habitants des pays chauds. Thomas Thorild, dans un poème judicieusement intitulé Norden (Le Nord) (1806), fait écho à cette conception lorsqu'il rédige différentes strophes dans lesquelles il célèbre son admiration pour les individus vivant dans les contrées nordiques. Je me permets d'en reproduire ici quelques-unes parmi les plus représentatives.

Mesen förakltig föraktar vårt Norden,

Friskhet och Frihet ej livar hans Bröst,

Vintern allt stärker, uppfriskar, förnyar .

Däremot se hur det går där i Söder,

Och du skall känna dig stolt i din Nord!

Se hur sig Fransmannen krummar och kröker

Men med Nordens Ätt kom Frihet och Rätt"7.

Puis, toujours dans ce mouvement d'opposition à l'eurocentrisme restreint, de nouvelles théories climatiques axées sur la productivité industrielle et l'exploitation des ressources naturelles se mêlent aux anciennes théories du goticisme dans la Suède du XVIIIe siècle 48 . C'est à une époque où pullulent les théories dites du nygöticism 
(nouveau goticisme), comme par exemple celle d'Eric Julius Biörner (1696-1750), que la théorie des climats de Montesquieu développée dans L'Esprit des lois (1748) fait son entrée en Suède. S'il est vrai que dans les textes de Pehr Wilhelm Wargentin (1717-1783)49 de la fin des années 1750 portant sur le climat, toute référence à la théorie des climats de Montesquieu est encore absente, on retrouve néanmoins dans la description de voyage de Carl Hårleman (1749-1750) un examen des thèses développées dans le livre XXIII de L'Esprit des lois50. Or, comme nous le savons tous, c'est principalement au livre XIV que Montesquieu introduit et présente la théorie des climats. II semble qu'Olof Dalin (1708-1763), dans Svea Rikes Historia : Ifrån des begynnelse til wåra tider (1750) [Histoire du royaume des Sveas : Des origines à aujourd'hui.] fut le premier en Suède à mentionner cette théorie de Montesquieu. II est important de noter qu'Olof Dalin, le Voltaire du Nord, n'était pas un nouveau venu en la matière car il avait déjà abordé, aussi tôt que 1744, la question de l'influence du climat sur les hommes 51 . Par conséquent, il est indéniable que la théorie des climats de Montesquieu avait de quoi plaire aux habitants des contrées nordiques, car contrairement à la théorie antique des climats où, comme nous l'avons vu, la zone septentrionale était perçue comme dégénérée, la théorie de Montesquieu reconnaît certaines vertus aux climats froids régnant dans les contrées nordiques. Dans une citation désormais classique, Montesquieu écrit :

L'air froid resserre les extrémités des fibres extérieures de notre corps; cela augmente leur ressort, et favorise le retour du sang des extrémités vers le cœur.

Il diminue la longueur de ces mêmes fibres; il augmente donc encore par-là leur force. L'air chaud, au contraire, relâche les extrémités des fibres, et les allonge; il diminue donc leur force et leur ressort.

On a donc plus de vigueur dans les climats froids. L'action du cœur et la réaction des extrémités des fibres s'y font mieux, les liqueurs sont mieux en équilibre, le sang est plus déterminé vers le cœur, et réciproquement le cœur a plus de puissance. Cette force plus grande doit produire bien des effets : par exemple, plus de confiance en soi-même, c'est-à-dire plus de courage; plus de connaissance de sa supériorité, c'est-à-dire moins de désir de la vengeance; plus d'opinion de sa sûreté, 
c'est-à-dire plus de franchise, moins de soupçons, de politique et de ruse. Enfin cela doit faire des caractères bien différents. Mettez un homme dans un lieu chaud et enfermé, il souftrira, par les raisons que je viens de dire, une défaillance de cœur très grande. $\mathrm{Si}$, dans cette circonstance, on va lui proposer une action hardie, je crois qu'on l'y trouvera très peu disposé; sa faiblesse présente mettra un découragement dans son âme; il craindra tout, parce qu'il sentira qu'il ne peut rien. Les peuples des pays chauds sont timides comme les vieillards le sont; ceux des pays froids sont courageux comme le sont les jeunes gens. Si nous faisons attention aux dernières guerres, qui sont celles que nous avons le plus sous les yeux, et dans lesquelles nous pouvons mieux voir de certains effets légers, imperceptibles de loin, nous sentirons bien que les peuples du nord, transportés dans les pays du midi, n'y ont pas fait d'aussi belles actions que leurs compatriotes qui, combattant dans leur propre climat, y jouissaient de tout leur courage 52 .

Cependant, le plus ardent défenseur suédois de la théorie des climats de Montesquieu est sans nul doute Jacob Fredrik Neikter (17441803), philosophe d'Uppsala suivant les brisées de Locke et combattant farouchement la philosophie rationaliste de Leibniz et Wolff. En octobre 1774, Neikter annonce ses couleurs lorsqu'il dénigre dans $D e$ Poësi Tragica les modèles poétiques (et artistiques) importés de la France. On rapporte même qu'il fut le premier à introduire l'œuvre de William Shakespeare en Suède, fait digne de mention si on connaît l'attrait qu'a exercé auprès des partisans du Sturm und Drang la figure insigne de ce grand dramaturge britannique. Dans une série de textes, tous écrits en latin, Neikter épouse et diffuse les thèses climatiques de Montesquieu en les adaptant toutefois à l'univers suédois. Parmi les textes les plus intéressants de Neikter mentionnons De efficacia climatum ad variam gentium indolem praecipue ingenia et mores I-XVI (1777-1797), Den gente Antiqua Troll, I-VI (1793-1796) et De Immutato per Culturam Locorum Climate, I-V (1795-1799). II est particulièrement important de mentionner pour notre propos que Thomas Thorild avait demandé à Neikter de présider sa défense pro exercitio du Critik öfver Montesquieu. Comment interpréter ce geste de Thorild? S'agissait-il d'une provocation envers les défenseurs de la théorie des climats de Montesquieu ou bien était-ce le désir de se 
frotter à une figure reconnue capable de lui opposer des arguments bien conçus qui motiva son choix? Nul ne le sait. Ce que l'on sait toutefois, c'est que Neikter déclina l'offre de Thorild, préférant sans doute rester loin de cette figure controversée des lettres suédoises. Neikter n'avait-il pas naguère vertement critiqué l'attitude peu cavalière de Thorild envers Kellgren dans le débat du début des années 1780 qui opposa ces deux titans? Carl Frängsmyr avance l'idée que Neikter, membre de la société Appolini Sacra - proche parente de la société Utile Dulci - n'avait guère apprécié le geste de mépris de Thorild envers la société Utile Dulci lorsque celui-ci refusa le second prix décerné pour son poème Passionerna. ${ }^{53}$ Mais revenons plutôt à cette soirée de Pâques 1788. En quoi le petit texte de Thorild est-il remarquable? Pourquoi l'histoire a gardé en mémoire cet événement, qui de prime abord semble si anodin?

Disons premièrement qu'une contestation ouverte de certaines thèses du Français avait de quoi attirer une horde de curieux...et d'adversaires, car, comme nous l'avons vu, les thèses climatiques de Montesquieu avaient trouvé en Suède un terrain propice pour se développer. Sans résumer l'ensemble de la critique thorildienne des thèses de Montesquieu, je me contente dans le cadre de cet article de mentionner uniquement la critique qu'il dirige à l'endroit de la théorie des climats.

Det inflyter. Men kan det därföre, enligt M. vara et ämne för Lagstiftning? Det inflyter; men så som Morgon, Middag och Afton, som klart och Mulet, som årstiderna, och lifvets 4 åldrar. Gör man Lagar för dessa? Climatets verkan är Nuance. Knapt skiljer den de fina och sköna Konsterna. Men Lagstiftning har at göra med Lif, Förstånd och Hjerta; med det hela och stora af Människan.

MONTESQUIEU är altid stor och sinrik : sällan vigtig och san.

Men i hvart Climat har Regeringen et och samma mål at söka, som är högsta Nationella Styrka och Sundhef54.

Thorild reconnaît certes une certaine influence du climat sur les individus. Mais, à l'instar de son collègue danois, Ludvig Holberg55, il s'empresse de souligner les limites et les insuffisances de la théorie des climats de Montesquieu. Loin de partager les conclusions de 
Montesquieu, basées sur un déterminisme strict établissant une corrélation directe entre le climat et la constitution d'un régime politique donné, Thorild réintroduit un élément universaliste en soutenant clairement que le but ultime (et peut être le seul) des régimes politiques est "d'accroître la puissance nationale et la santé." On peut également lire en filigrane dans la position de Thorild, la critique qu'avait adressé Helvetius à Montesquieu dans De l'Esprit (1758). On a certes reproché à Thorild, dans sa contestation des thèses de Montesquieu, d'avoir davantage promu les thèses qui lui étaient chères que d'avoir répliqué d'une façon systématique aux idées du baron français 56 . Thorild concède bien à Montesquieu une certaine influence du climat, mais cela devient uniquement une influence anodine parmi un faisceau d'éléments inextricables concourant à la détermination de la législation. Par conséquent, la détermination stricte de la législation par le climat que désire introduire Montesquieu est grandement diluée par Thorild et, comme corollaire, toute forme de relativisme semble exclue. Là où chez Montesquieu - et par la suite chez Herder - le principe du relativisme devient un élément indissociable d'une compréhension du monde dans son devenir, règne chez Thorild une conception moniste de l'univers conditionnée par son panthéisme.

Même si à d'autres moments de sa production littéraire il arrive à Thorild de mentionner 57 le nom de Montesquieu (mais pas nécessairement lié quant à la théorie des climats), jamais il n'est revenu sur son jugement concernant la théorie des climats. Nous possédons donc maintenant un élément nous permettant de comparer les positions respectives de Thorild et de Herder. Passons maintenant à ce second élément : quelle opinion Herder avait-il de la théorie des climats de Montesquieu, à la même époque?

\section{Herder et Montesquieu}

Guère choyée par la typologie des trois zones climatiques - car l'Allemagne a été longtemps relégué au rang des pays exclus de la zone des pays tempérés jouissant des privilèges attribués au climat tempéré - la théorie des climats était cependant bien connue dans les pays germanophones d'un aréopage d'intellectuels de renom. Parmi ceux-ci nous pouvons nommer Johann Joachim Winckelmann (1717-1768), Gotthold Ephraim Lessing (1729-1781), Albrecht von Haller (1708-1777), Christoph Martin Wieland (1733-1813), Johann Georg Hamann (1730-1788), Johann Gottfried Herder (1744-1803), 
Immanuel Kant (1724-1804) et Georg Forster (1754-1794)58. Sans tenter d'exposer dans toute sa complexité la relation de Herder envers la théorie des climats de Montesquieu, nous désirons nous concentrer sur la lecture herdérienne de cette thèse à une époque recoupant celle où Thorild s'y est intéressé.

Herder a connu très tôt différentes théories tentant d'établir une corrélation entre le climat et l'influence que celui-ci exerce sur les êtres humains. Une présence pratiquement continue de la théorie des climats dans les différents écrits de Herder n'occulte cependant pas le fait que le philosophe allemand demeure toujours conscient de la difficulté et de la complexité du rapport existant entre le climat et les individus. Herder a d'ailleurs écrit dans Idées pour une philosophie de l'Histoire : «nous sommes une argile malléable dans la main du climat; mais ses doigts sont si habiles à produire des formes diverses, et les lois qui lui résistent si variées, que peut-être seul le génie humain serait capable de mettre en équation le rapport de toutes ces forces". Cela, sans aucun doute, témoigne de la complexité de la question.

Depuis quand l'influence de la théorie des climats de Montesquieu s'est-elle fait sentir chez Herder? On sait évidemment qu'il a suivi à Königsberg les leçons de Kant sur la géographie où il était déjà question de la théorie des climats, mais c'est surtout la lecture d'un livre de Winckelmann, Gedanken über die Nachahmung der griechischen Werke (1755) (Réflexions sur l'imitation des œuvres grecques en peinture et en sculpture), et de l'Introduction à l'Histoire de Danemark de Paul-Henri Mallet (1730-1807) que les thèses de Montesquieu sur le climat captent son attention.

II est vrai que l'influence des climats demeure un thème constant de la production littéraire de Herder. Par exemple, dans un texte intitulé Über den Fleiß in mehreren gelehrten Sprachen (1764) ( $D u$ zèle en plusieurs langues savantes) Herder reprend un lieu commun, hérité de la philosophie grecque, lorsqu'il écrit que «les centaines de langues furent crées en conformité avec les mœurs et le climat d'une centaine de nations ${ }^{59}$ '. Mais c'est véritablement dans un texte de 1766, Ist die Schönheit des Körpers ein Bote von der Schönheit der Seele? (La Beauté du corps est-elle messagère de la beauté de l'âme?) que l'on sent poindre davantage la théorie de l'influence des climats sur les aspects physiques des individus et plus particulièrement sur la mentalité des êtres qui le subissent. Pour 
appuyer son argument, Herder n'hésite pas d'ailleurs à faire explicitement appel à Montesquieu, Montagne (sic) et Beaumelle60.

Les textes jalonnant la production littéraire de Herder portent constamment la marque de la théorie des climats de Montesquieu, mais de façon différente. Toutefois, c'est au cours des années 1770 que Herder couple la théorie des climats à sa conception de l'histoire. On peut dire dès lors que la théorie des climats de Montesquieu assure le substrat théorique à partir duquel Herder parvient à concevoir une philosophie de l'histoire et parvenir à une conception relativiste de l'humanité.

Comme le note Fink:

La situation, revalorisée par Herder dans une visée patriotique, ressemblait énormément à celle dépeinte par Montesquieu, mais chez Herder le critère politique de la liberté est remplacé par celui vitaliste de la vie et de la force. A l'époque de Bückenburg, Herder maintenait ainsi les éléments essentiels de la théorie des climats, mais celle-ci est relativisée en raison d'une confrontation avec l'histoire et soumise au besoin à sa perspective biblique, vitaliste ou patriotique ${ }^{61}$.

Comme on peut s'y attendre, la théorie des climats occupe également une position centrale dans un de ses principaux textes des années 80 , les ldées sur la philosophie de l'histoire de l'humanité62.

Dans ce texte, à l'instar de Montesquieu mais également de Dubos, Herder démontre à grand renfort d'exemples l'influence qu'exerce le climat sur la mentalité des peuples. Comme nous avons choisi comme stratégie de comparer la perception qu'avait Thorild de la théorie des climats de Montesquieu en 1788 à la perception qu'en avait Herder à la même époque, je crois qu'il est important de signaler ici une ressemblance troublante entre les deux auteurs. D'une part, comme nous l'avons déjà mentionné, Thorild avait soutenu que les climats influencent [inflyter] mais que cette influence se fait tout en nuances. Herder, d'autre part, soutient ceci : «Le climat ne contraint pas, mais il incline ${ }^{63}$ ”. Que penser de cette étonnante similitude dans la formulation de l'influence putative du climat sur les individus? On reconnaît certes, de part et d'autre, que le climat exerce une influence mais, en même temps, on tente de limiter l'influence de celui-ci, soit - chez Thorild - en recherchant un principe universel de justice ou bien - chez Herder - en liant la thèse du déterminisme 
climatique à une conception dynamique et relativiste de l'histoire. Or c'est précisément dans l'atténuation du déterminisme climatique que Thorild et Herder se distinguent. Chez Thorild le relativisme qu'induit l'influence du climat sur les êtres humains qui le subissent est contré par la recherche d'un objectif universel : "accroître la puissance nationale et la santé" alors que chez Herder on recherche plutôt à intégrer l'influence du climat dans une dimension historique complexe. Bien qu'à certains égards les positions de Thorild et de Herder semblent parfois indiscernables, voire insaisissables, force est de constater que leur manière respective d'aborder les thèses de Montesquieu concernant la théorie des climats diffèrent grandement l'une de l'autre.

\section{Conclusion}

"Thorilds system kan parafraseras men inte prövas....»64. Cette évaluation lapidaire, mais hélas combien vraie, de Horace Engdahl constitue certes un élément rendant difficile, complexifiant, toute "compréhension», puis par la suite toute "comparaison» de la philosophie de Thomas Thorild. Reconnu pour le caractère hautement sibyllin de son écriture, cette particularité s'applique également à Herder. Les problèmes émanant de la comparaison de deux systèmes de pensé aussi abstraits et énigmatiques ne simplifie guère la tâche. Pour contourner les problèmes intrinsèquement liés au style d'écriture de ces deux auteurs, nous avons tenté de réduire au maximum les difficultés militant contre une telle comparaison en isolant au préalable un élément leur étant commun. L'élément choisi a été la théorie des climats de Montesquieu. Or, même si on note une certaine ressemblance dans la manière de faire de la philosophie et de solutionner des problèmes communs, il n'en demeure pas moins que Thorild ne doit pas être perçu comme un pâle imitateur de Herder. Thorild adopte plutôt une attitude très critique envers le déterminisme strict qu'induit la théorie des climats de Montesquieu. Cette attitude critique semble complètement disparaître chez Herder qui, au contraire, assume consciemment les implications relativistes qui découlent de la théorie des climats de Montesquieu.

Que nous apprend de fait la comparaison entre Thorild et Herder en ce qui concerne la théorie des climats de Montesquieu? Devonsnous voir dans cela une réfutation de l'hypothèse de Cassirer? Nous possédons pour l'instant trop peu d'éléments pour nous permettre de réfuter d'une façon "catégorique» l'hypothèse de Cassirer. 
Cependant, le contre-exemple que nous offre leur conception respective de la théorie des climats de Montesquieu est "suffisant»pour que l'on doute de la validité de l'hypothèse de Cassirer. La reconnaissance d'un tel doute, aussi minime soit-il, nous incite à chercher d'autres éléments tendant à valider la thèse de l'indépendance (entière et totale) de Thorild envers Herder. La reconnaissance de l'indépendance philosophique de Thorild vis-à-vis de Herder ne signifie toutefois pas qu'il faille occulter, ou au pis nier, l'influence du philosophe allemand sur le Suédois. Tout au contraire, l'assignation de leur mérite respectif constitue le seul et unique moyen de rendre justice à leur grandeur respective.

Prenant cela en considération, il serait intéressant d'analyser plus en profondeur sous quelle forme le mouvement philosophique du Sturm und Drang s'est développé chez Thorild. L'histoire du développement de ce mouvement en Suède, principalement sous l'impulsion de Thorild - son véritable fer de lance - , nous apprend que le contact avec ce courant d'expression allemand s'est fait davantage par l'entremise de l'école dite de Göttingen65, dont Ludwig Hölty (1748-1776), Johann Henrich Voss (1751-1826), Matthias Claudius (1740-1815) et Gottfried August Bürger (1747-1794) comptaient parmi les membres les plus éminents. C'est cependant au contact du musicien allemand Joseph Martin Krauss (1756-1792), arrivé en Suède à l'âge de 22 ans, et du poète suédois Carl Stridsberg (1755-1819) que Thorild découvre et, dans un second temps, s'entiche du mouvement du Sturm und Drang. Si on peut soutenir, sans risque de se tromper, que Thorild est le premier représentant suédois du mouvement du Sturm und Drang, il ne faudrait cependant pas oublier que cette influence lui a été transmise par des représentants appartenant à l'école de Göttingen. Cet élément tend à démontrer que l'attrait esthétique et philosophique ressenti par Thorild pour ce mouvement littéraire ne découle nullement de Herder - lequel est plutôt associé à la conception weimarienne du Sturm und Drang - mais provient plutôt d'une autre source témoignant d'une influence différente; influence qui d'une certaine manière s'enracine plus profondément dans l'âme suédoise. S'il est vrai que les philosophies respectives de Thorild et de Herder présentent bien des ressemblances troublantes, le fait demeure que c'est davantage vers des compatriotes suédois ('Allemand Krauss, dénommé le Mozart suédois, passera la plus grande partie de sa vie en Suède) que se tourne Thorild lorsqu'il cherche des modèles littéraires à initier. 
Prenant en considération ces faits peu ou mal connus, que penser alors de la tentative de Cassirer de faire de Thorild une sorte de Herder suédois, une pâle copie du maître? S'il est vrai qu'il existe une étonnante ressemblance dans leur critique respective de la Critique de la raison pure, il faut davantage percevoir cela comme une coïncidence tenant davantage de l'adhésion à un courant artistique global, présentant des nombreux traits communs et, partant, présentant de grandes ressemblances tant au niveau philosophique que stylistique, plutôt que comme le désir patent de Thorild d'imiter le philosophe allemand. Seule une nouvelle analyse en profondeur de l'Archimetria et de la Metakritik, dégagée de toute préconception redirigeant trop rapidement l'interprétation de leur philosophie respective en invoquant l'existence et la présence d'un "effet miroir» où les images de Thorild et de Herder se refléteraient mutuellement, nous permettrait d'attribuer la valeur respective de ces œuvres de maturité.

En attendant, une comparaison entre ces deux auteurs sur un aspect précis de la théorie de Montesquieu nous paraît alors suffisante pour douter du bien-fondé de l'hypothèse de Cassirer. Par conséquent, l'apparition d'un doute quant à la parenté philosophique de Thorild et Herder au milieu des années 1780 constitue un élément suffisant nous permettant de croire qu'il faille soumettre la thèse de Cassirer à un nouvel examen. Une étude plus approfondie des thèmes propres à ces auteurs respectifs nous démontrerait l'indépendance de l'un par rapport à l'autre, mais ce travail demeure toujours à faire.

\author{
Roch Duval \\ Université de Montréal \\ Département de linguistique \\ et de traduction \\ roch.duval@umontreal.ca
}

1. Jean-Paul Richter, Cours Préparatoires d'Esthétique. Cité dans Pierre Pénisson, La raison dans les peuples. Paris, Éditions du Cerf, 1992, p. 11.

2. "Dans son cerveau régnait un monde de chaos, où ne luisait ni le soleil, ni même l'étoile du matin, uniquement un météore éclatant et se dispersant". Strophe tirée du poème "Sång den 5 april 1836". Cité dans Teddy Brunius (dir.), Anders Karitz, Skrifter $i$ urval 2, Thorild och hans filosofi, Fören. för filosofi och specialvetenskap, Uppsala, Solna, 1977, p. 160. (Les traductions, sauf indication contraire, sont les miennes). 
3. Il est intéressant de noter toutefois que le livre était dédié à Herder et Jean Paul, autre figure symbolique du Sturm und Drang. Thorild y exprime d'ailleurs sa grande admiration en écrivant : “Herder! Genius der Humanitätl» (Herder! Génie de l'humanité1).

4. "Mon mari tient en haute estime ce livre; il en lit des passages tous les jours". Von und an Herder. Ungedruckte Briefe aus Herders Nachlaß. H. Düntzer et F. G. von Herder. Bd. 1, Leipzig, 1861, p. 267.

5. Un long article dans l'Allgemeine Literaturzeitung avait voué aux gémonies le Maximum seu Archimetria. On y soutenait, entre autres, que l'Archimetria, malgré son feu intérieur et la richesse de son contenu, n'offrait somme toute que des "bulles de savon philosophiques".

6. Compte rendu reproduit dans le volume 20 des œuvres complètes de Herder. Sämtliche Werke, Bernhard Suphan (éd.), Bd. 20, 1880, p. 367-371.

7. "L'auteur de cette Archimetria est Thomas Thorild, poète suédois, et le premier a avoir introduit dans sa patrie un accent poétique inspiré de Klopstock et de Young."Cité dans Per Daniel Amadeus Atterbom, Svenska siare och skalder. (Prophètes et poètes suédois), Del 5, Uppsala, 1849, p. 288.

8. "Ein Philosoph in starren Schulverstande dieses Wortes kann er freilich nicht genannt werde, und er selbst wäre der Letzte, der dieses Prädikat beansprucht." (On ne pouvait le nommer un philosophe au sens académique du terme, et il était certes le dernier à vouloir revendiquer ce terme) Carl Siegel, Herder als Philosoph, J.G. Cotta , Stuttgart et Berlin, 1907, p. XIII.

"Aber war Thorild überhaupt ein Philosoph, und kann sein Werk mit rein-philosophischen Maßstäben gemessen werden?" (Mais après tout, Thorild était-il un philosophe et peut-on mesurer son œuvre à l'aune de critères philosophiques purs?) Ernst Cassirrer, Thorilds Stellung in der Geistesgeschichte des achtzehnten Jahrhunderts. Wahlström \& Widstrand, Stockholm, 1941, p. 5. Rassurons ici tout de suite le lecteur, la réponse de Cassirer est positive.

9. Malmström, Györki et Sjögren (dir.) Bonniers svenska ordbok, Bonniers, Stockholm, 1999.

10. Voir, Thomas Thorild, Att följa ögonblicken. Texter i urval. Introduction et notes de Horace Engdahl, Svenska Akademien, Bokforlaget Atlantis, Stockholm, 2000, p. 359.

11. Il est inutile de toutes les mentionner ici. Cela débuta avec le célèbre homme de lettre Erik Gustaf Geijer qui publia en 1820 une étude intitulée Thorild. Tillika en filosofisk eller ofilosofisk bekännelse pour se poursuivre jusqu'à la monumentale biographie de Stellan Arvidson, parue en 1989 et en 1993. Stellan Arvidson, Passionernas diktare, Thorild I, 1989 et Harmens diktare. Thorild II, Carlssons, Stockholm, 1993.

12. "Aber woher stammt diese ideelle Gemeinschaft zwischen Thorild und Herder, und wann hat sie sich zuerst geknüpft?" "(Mais d'où provient donc cette correspondance conceptuelle entre Thorild et Herder, et quand s'est-elle formée?». Ernst Cassirer, "Thorild und Herder», Theoria (1941), vol., VII, no. 2, p. 75-92, p. 75.

13. Ernst Cassirer, "Thorild und Herder», Theoria (1941), vol., VII, no. 2, p. 75-92 et Thorilds Stellung in der Geistesgeschichte des achtzehnten Jahrhunderts Wahiström \& Widstrand, Stockholm, 1941.

14. Alf Nyman, Exilens Filosofi. En studie $i$ Thomas Thorilds Arkimetrai. [Philosophie de l'exil. Étude sur l'Archimetria de Thomas Thorild], Lund, 1956, Lunds Universitets Årsskrift, N.F., Avd 1, Bd. 52, Nr 4., 1956. 
15. Cette influence devient encore plus manifeste lorsqu'on prend en considération le rôle qu'a joué Lessing chez Thorild et Herder. Voir Adolf Häckermann, «Thomas Thorild, der skandinavische Lessing. Ein schwedisch-deutsches Lebens- und Charakterbild.”, Sonntags-Blatt, Jahrg. 8 (1883), no, 4-9, p. 11-18.

16. "Dass Thorild diese Schrift gelesen hat, dafür haben wir freilich keinen direkten Beweis." ( $\dot{A}$ vrai dire, nous n'avons pas de preuves directes que Thorild a lu ce texte.) Cassirer, "Thorild und Herder», op. cit., p. 77.

17. Thomas Thorild, "Critik öfver Montesquieu, under juris professorens doctor Anders Hernbergus presiderande, försvarad den 22 mart. 1788."Stellan Arvidson (dir.) Thorild samlede verke, hefte 4, p. 279-283.

18. Le lecteur averti aura compris le clin d'œil qui est fait au célèbre poème philosophique de Thorild intitulé Passionerna (Les passions) (1781). L'origine du différend opposant Thorild à Kellgren remonte au moment où le poème de Thorild ne remporta pas le prix offert par la société Utile Dulci, présidée alors par Kellgren.

19. Poète, dramaturge, traducteur et philosophe des Lumières. Kellgren fur le premier président de la Svenska Akademien (1786), calquée sur le modèle de l'Académie française. II existe depuis peu une édition critique d'une grande qualité des œuvres de Kellgren. Voir Carina et Lars Burman (éd.) avec une introduction de Torgny Segerstedt, Johan Henric Kellgren. Skrifter I Poesi och prosa, (Johan Henric Kellgren. Écrits I. Poésie et prose), Atlantis, Stockholm, 1996. (Svenska klassiker).

20. Ce représentant des Lumières fut également le secrétaire personnel du roi Gustave III. À la mort de Kellgren il devient le principal opposant de Thorild. Leurs chemins se croisèrent à Greifswald.

21. À cet effet, voir de Bernhard von Beskow, Om den estetiska betydelsen af Thorilds strid emot Kellgren och Leopold (Sur la signification esthétique de la querelle de Thorild contre Kellgren et Leopold), Svenska akademiens handlingar ifrån år 1796 D. 48. Notons que vers la fin de sa vie Kellgren avait pris ses distances par rapport au Classicisme français et le roi Gustave III.

22. Voir Svensk Sturm und Drang. Thomas Thorild. Bengt Lidner i urval. (Le Sturm und Drang suédois. Sélection de textes de Thomas Thorild et de Bengt Lidner FIB lyrikklubb, Stockholm, 1957). Bien qu'il existe un équivalent suédois de l'expression allemande Sturm und Drang (storm och jäsning), il est de coutume de maintenir l'expression en allemand.

23. Il est intéressant de mentionner, à cet égard, que c'est une citation d'Edward Young tirée de "Conjectures on original composition", qui chapeaute le poème Passionerna de Thorild. "Born Originals, how comes it to pass that we die Copies?"

25. Horace Engdahl note cela avec justesse. "Han fick anhängare bland de unga, men obetydliga, han brände chansen att vinna tillträde till maktens cirklar, hans litterära och filosofiska motståndare gick segrande fram." "ll [Thorild] eu des supporters parmi les jeunes, mais il brûla inutilement ses chances d'accéder aux sphères du pouvoir, ses opposants littéraires et philosophiques ayant su le devancer." p. VII.

25. Le 12 avril 1785 Thorén modifia son nom pour celui de Thorild. Ce changement est significatif de l'état d'esprit animant le philosophe suédois à cette époque. Thorild provient des mots suédois Thors et eld (le feu de Thor). Arvidsson écrit : "Skalden hade ju 1785 ändrat sitt namn från Thorén till Thorild, som bör betyda Thors eld. Identifikationenen mellan skalden själv och Thor är i dikten understruken :

Thors gnista jag kände 


\section{Och kände hans Harm.}

(Comme on le sait, le poète a changé en 1785 son nom de Thorén en Thorild, ce qui signifie le feu de Thor. L'identification entre le poète et Thor est soulignée dans un poème : Je connaissais l'étincelle de Thor/ et je connaissais son indignation). Notons que dans les autres langues scandinaves [norvégien et danois] "feu»se dit «lld".

Arvidsson (1993), op. cit., p. 304.

26. Un monument commémoratif a été érigé à cet endroit en 1928.

27. "Le séjour dans la maison du directeur fut vraisemblablement significatif pour le développement intellectuel de Thorild et pour son engouement pour les études."Stellan Arvidsson, (op. cit.), p. 80.

28. On peut également conjecturer que Thorild a connu les œuvres de l'orientaliste David Michaelis par l'entremise de l'enseignement de Gothenius, car ce dernier avait été son élève à Göttingen.

29. Les biographes possèdent très peu d'information sur ce second séjour. "Tre och en halv månader varade hans andra Göteborgssejour, om vilken vi inte har den minsta upplysning." (Nous avons un nombre limité d'informations sur son second séjour d'une durée de trois mois et demi à Göteborg.) Voir Arvidsson, (op. cit.) p. 269.

30. (C'est alors qu'il était étudiant à Lund qu'il fit connaissance des nouveaux courants de pensée, en partie révolutionnaires, provenant de l'Europe, principalement de la France, de l'Angleterre et de l'Allemagne. Lorsqu'il quitta la ville universitaire, à la fin de l'année 1780, il apportait dans ses bagages une nouvelle vision du monde antichrétienne, de nouvelles conceptions philosophiques et une nouvelle conception littéraire, influencée par le Werther de Goethe. [...] Son séjour à Lund fut consacré à faire progresser ses propres études.) Arvidsson, op. cit., p. 333.

31. L'intérêt de Thorild pour les questions religieuses fut motivé par les lectures qu'il fit à cette époque. Mentionnons en particulier un livre de Hermann Samuel Reimarus, Die vornehmsten Wahrheiten der natürlichen Religion (1754) et un ouvrage de Charles Bonnet, Recherches philosophiques sur les preuves du christianisme. Voir le texte de Thorild intitulé En pantheists anmärkningar vid Reimarus. Voir également l'esquisse qu'il a rédigée à cette époque (1780) et intitulée Min religion (Ma religion).

32. Dans une lettre datée du 12 octobre 1780 et destinée à Thorild, Sven Erland Heurlin désigne Lund comme le "siège de l'imbécillité" (dumhetens hemvist). L. Weibulll (éd.). Thorilds bref, p. 15.

33. Une des nombreuses îles sur lesquelles est bâti Stockholm.

34. "Serein et imprégné par le paysage, je t'écris assis près de ma fenêtre ouverte et tous mes sens se délectent de la beauté de Dieu et respirent son être". Thorilds bref, op. cit., p. 47.

35. En 1784, Elis Schröderheim le désignera comme le «förryckta Magistern»(le professeur fou). Voir Karitz, op. cit., p. 160 et Arvidsson (1993), op. cit. p. 107.

36. Le 22 mars 1788.

37. On rapporte que Gustave III avait été favorablement impressionné par Thorild. Notons que lorsqu'il se dévoile à Herder, Thorild mentionne l'estime que le roi lui portait. 
38. "Nulle doctrine dans L'Esprit des Lois n'a attiré plus l'attention que celle de l'influence du climat sur les hommes."Robert Shackhelton, Montesquieu : A Critical Biography, Londres, 1961, p. 302.

39. Hippocrate. Airs, eaux, lieux, Rivages Poches, Paris, 1996.

40. Aristote, Politique, Septième livre. Traduction de Jean Aubonnet. Les Belles Lettres, Paris, 1986 (Budé Série Grecque)

41. Cette théorie des trois climats se perpétua tout le long du Moyen Age. Même lbn Khaldun, dans le al-Muqaddima s'en inspire pour introduire sa théorie des sept zones climatiques. La théorie de Jean Bodin, esquissée dans Methodus ad facilem historiarum cognitionem (1566), si influente au siècle Classique, est une descendante directe de la théorie aristotélicienne des trois climats.

42. Faisant d'une manière honteusement réductrice de la France, de l'Italie et de la Grèce les uniques lieux du bon goût et, par ricochet, de la culture au sens louable du terme.

43. Jean-Baptiste Dubos (Abbé Du Bos). Réflexions critiques sur la poësie et sur la peinture; préface de Dominique Désirat [1755]. Paris: École Nationale Supérieure des Beaux-Arts, 1993.

44. Voir de Roger Mercier, "La théorie des climats des Réflexions critiques à L'Esprit des lois", Revue d'histoire littéraire de la France, (janvier, mars et avril, juin 1953), pp. 17-37 et 159-175.

45. Olaus Magnus, Historia om de nordiska folken, I-IV, Stockholm, 1976.

46. "större och manhaftigare både till Kropp och Sinne". Olof Rudbeck, Atlantican, I-V, Lychnos-biliothek 2: 1-2:5 (Uppsala, 1937-1950), p. 57.

47. Le couard méprise avec dédain notre Nord

La fraîcheur et la liberté ne vivent pas dans son sein,

L'hiver fortifie tout, revigore, régénère.

Regarde au contraire comment il en va dans le Sud, et tu te sentiras fier dans ton Nord!

Regarde comment les Français rampent et sont serviles

.....

Mais avec la dynastie nordique est venue la liberté et la justice.

Thomas Thorild, Att följa ögonblicken, op. cit. p. 141.

48. Pour illustrer les différentes théories ayant cours à cette époque je m'inspire abondamment du merveilleux livre de Carl Frängsmyr, Klimat och karaktär. Naturen och människan i sent svenskt 1700-tal. [Le climat et le caractère. La nature et l'homme en Suède vers la fin du XVIIIe siècle], Bonniers, Stockholm, 2001.

49. Pehr Wilhlem Wargentin, "Anmärkningar om Climaters Skiljaktighet» (Remarques sur les différences climatiques) Kungliga Vetenskapsakademiens Handlingar (1757); "Anmärkningar om Svenska Climatet» (Remarques sur le climat suédois), ibid. 1757; "Jämförelse Imellan Svenska och Franska Climaterna, samt tvänne andre Sydligare" (Comparaison entre les climats suédois et français avec vingt autres pays plus au sud), ibid. 1758.

50. Carl Hårleman, Dag-Bok öfwer En ifrån Stockholm igenom åtskillige Rikets Landskaper gjord Resa år 1749. (Journal sur un voyage de Stockholm vers différentes provinces du Royaume fait en 1749), 1749-1751. II y est notamment 
question de la consommation de poisson des peuples côtiers et l'influence de ce régime sur leur tempérament.

51. Olof Dalin. Påminennelse-Tal öfver Kongl. Vetenskaps Academiens medlem ÄrkeBiskopen Herr Doctor Eric Benzelius. Stockholm, 1744.

52. Montesquieu, De l'esprit des lois. p. 613.

53. Frängsmyr, op. cit., p. 85.

54. Cela influence. Mais cela peut-il être comme le soutient Montesquieu une cause de législation? Cela influence, mais tout comme le font le matin, le midi et le soir, un ciel dégagé ou couvert, les saisons et les quatre âges de la vie. Fait-on pour autant des lois pour cela? L'influence du climat s'exerce en nuance. Cela permet à peine de distinguer les beaux-arts. Mais la législation concerne la vie, la raison et le cœur, tout l'ensemble de ce qui constitue un homme.

Montesquieu est toujours grand et ingénieux, mais il est rarement souple et vrai. .....

Mais sous chaque climat les régimes politiques poursuivent le même objectif, soit accroître la puissance nationale et la santé. Thorild, op. cit. heft 4, p. 292.

55. Voir Fredrik Bajer, Montesquieu i Holbergs og Thorilds omdeme (Monstesquieu selon Holberg et Thorild),Stockholm, 1879

56. Arvidsson (1993), op. cit., p. 393.

57. A l'instar de la distinction classique, il s'agit toujours uniquement de mention et jamais d'usage des théories de Montesquieu.

50. Voir Gonthier-Louis Fink, "Von Winckelmann bis Herder. Die deutsche Klimatheorie in europäischer Perspektive." dans Gerhard Sauder (dir.), Johann Gottfried Herder 1744-1803. Felix Meiner Verlag, Hamburg, 1983, p. 156-176.

59. "...und es schussen sich tausend Sprachen nach dem Klima und den Sitten von tausend Nationen."Suphan, I, p. 1.

60. SW I, p. 46. II s'agit bien entendu de Laurent Angliviel de La Beaumelle (17261773).

61. Das Bild, das Herder in patriotischer Intention aufwertete, ähnelte zwar demjenigen, das Montesquieu vermittelte, aber bei Herder machte das politische Kriterium der Freiheit dem vitalistischen von Leben und Kraft Platz. So behielt Herder in der Bückenburger Zeit zwar die wesentlichen Elemente der Klimatheorie bei, relativierte sie jedoch durch die Konfrontation mit der Geschichte und ordnete je nach Bedarf sie seiner biblischen, seiner vitalistischen oder patriotischen Perspektive unter. Fink, op. cit. p. 172.

62. Idées sur la philosophie de l'histoire de l'humanité, trad. E. Quinet, éd. M. Crépon, Presses Pocket, 1991 (Agora. Les Classiques).

63. SW XIII, p. 273. "Das Klima zwingt nicht, sondern es neigt».

64. "On peut paraphraser le système de Thorild,mais on ne peut le prouver». Engdahl, op. cit. p. VII.

65. Le Hainbund, ou l'école de Göttingen, est une variante du Sturm und Drang accordant une grande place à la poésie de Klopstock et à la musique de Gluck. 